\title{
Resultado del tratamiento con Lithostar Modularis de Siemens de 1500 pacientes con litiasis única
}

\author{
Vega Vega A, de Blas Gómez V, Guerreiro González R, Gallo Rolanía FJ. \\ Servicio de Urología. Complejo Hospitalario de León.
}

Actas Urol Esp. 2008;32(5):546-551

\begin{abstract}
RESUMEN
RESULTADO DEL TRATAMIENTO CON LITHOSTAR MODULARIS DE SIEMENS DE 1500 PACIENTES CON LITIASIS UNICA

Objetivos: Valoración de la eficacia de la litotricia extracorpórea en litiasis urinaria con el litotritor Lithostar Modularis de Siemens.

Pacientes y Métodos: de junio del 2000 a Septiembre de 2006 se trataron 1.500 pacientes, $62 \%$ varones y 38\% mujeres, con edades comprendidas entre 15 y 89 años, media 53 años, en la Unidad de Litotricia de León. Los datos se analizaron de forma retrospectiva. En el $56 \%$ de los enfermos la localización de la litiasis fue izquierda. En el uréter, el $55 \%$ de las litiasis, la localización más frecuente fue proximal (420 pacientes) y la localización más frecuente renal fue en la pelvis (345 pacientes). El tamaño medio de las litiasis fue de 1,3 cm $(1,01 \mathrm{~cm}$ en uréter y $1,7 \mathrm{~cm}$ renal). Todos los pacientes se trataron con sedación con alfentanil y midazolan.

Resultados: La media de sesiones por litiasis fue de 1,5 (1,4 en las ureterales y 1,7 en las renales) La tasa de retratamiento fue de $33 \%$ (28\% en el caso de las litiasis ureterales y $39 \%$ en las renales. La eficacia acumulada tras 2 sesiones fue de $86 \%$. El $22 \%$ precisó maniobras auxiliares (el $24 \%$ de las ureterales y el $18 \%$ de las renales). El porcentaje de pacientes libre de litiasis fue del $91 \%$ (97 \% en el caso de las ureterales y el $82 \%$ en el caso de las renales).

Conclusiones: El tratamiento de las litiasis urinarias mediante litotricia con el Lithostar Modularis de Siemens muestra unos buenos resultados, con más de un $90 \%$ de los pacientes libres de litiasis, aunque con un 33\% de retratamientos y más de un $20 \%$ de los pacientes precisan maniobras auxiliares.
\end{abstract}

Palabras clave: Litotricia extracorpórea. Litiasis renal.

\section{ABSTRACT \\ SINGLE CENTER EXPERIENCE WITH SIEMENS LITHOSTAR MODULARIS FOR TREATMENT OF 1500 SOLITIARY URINARY STONES}

Objectives: the aim of the study was to evaluate the results of extracorporeal shock wave lithotripsy (ESWL) treatment with Siemens Lithostar Modularis in urinary stones.

Patients and Methods: from may 2000 until September 2006, 1500 patients, $62 \%$ men and 38\% women, ages 15 to 89 years (mean age 53), underwent ESWL as treatment of urinary stones at the Lithotripsy Unit of León. Data were analized retrospectively. More than half (56\%) had left urinary stones. The most frequent ureteral localization (55\%) was proximal (420 patients), and the most frecuent renal was renal pelvis (345 patients). The mean stone diameter was $1.3 \mathrm{~cm}$. (1.01 ureteral and 1.7 renal). All patients received ESWL-session under i.v. analgesia with alfentanil and midazolam.

Results: Mean number of sessions per lithiasis was 1.5 (ureteral stones 1.4 and renal stones 1.7). The retreatment rate was $33 \%, 28 \%$ for ureteral stones and $39 \%$ for renal stones with a cumulative success of $86 \%$ with two sessions. $22 \%$ of the patients required auxiliary manouvers, $24 \%$ of the patients with ureteral stones and 18 $\%$ of the renal stones patients. Stone free rate was $91 \%, 97 \%$ in ureteral stones and $82 \%$ in renal stones.

Conclusions: treatment of urinary stones with Siemens Lithostar Modularis showed good results, with a stone free patient rate of more than 90\%, although $33 \%$ of the patients need multiple ESWL treatment sessions and $20 \%$ of the patient required auxiliary procedures.

Keywords: Extracorporeal shockware lithotripsy. Renal calculi. 
$\mathrm{L}$ a litotricia extracorpórea por ondas de choque es el tratamiento de elección de la mayoría de los cálculos urinarios. La aplicación de las ondas de choque para el tratamiento de las litiasis urinarias fue usada por primera vez en 1982 por Christian Chaussy $^{1}$. Desde entonces se ha producido una gran evolución en los aparatos de litotricia extracorpórea con diferentes modificaciones en la generación de las ondas de choque, el tamaño del punto focal, el método de acoplamiento del cabezal del aplicador de ondas y de los métodos de localización de la litiasis. Todos estos cambios repercuten en la capacidad de fragmentación de los litotritores ${ }^{2}$.

$\mathrm{El}$ generador de ondas de choque puede ser electrohidraúlico, electromagnético y/o piezoeléctrico. Para que se produzca la fragmentación de las litiasis se necesita que se concentre la energía en un punto concreto, esto se consigue mediante la focalización de las ondas de choque. Cuando no se focalizan, sólo se produce la fragmentación empleando energías muy altas, lo cual favorece que se produzcan lesiones en los tejidos circundantes, aumentando el riesgo de efectos secundarios. La focalización de las ondas se consigue mediante lentes acústicas de reflexión, y depende de 2 variables: la apertura del foco y del área focal. La apertura de la onda de choque depende de la anchura del reflector y de la superficie corporal que atraviesan las ondas de choque hasta llegar a la litiasis. En general una mayor apertura se asocia con menor dolor. El área focal es el volumen dentro del cual se concentran las ondas de choque, es donde se produce la fragmentación de la litiasis. A mayor área focal la localización es menos precisa y necesita más anestesia. Cuando tenemos una menor área focal, la localización es mejor y las necesidades de anestesia menor, las máquinas de litotricia actuales han evolucionado en esta dirección. Los otros dos factores que han experimentado modificaciones en el diseño de los nuevos aparatos de litotricia son el método de acoplamiento, que permite el paso de la energía sin una pérdida significativa de la misma, este ha pasado de métodos de inmersión a sistemas secos, más cómodos para el paciente, y otro factor modificado es el método empleado para la localización de la litiasis, en aras de evolución hacia conseguir una mejor imagen que favorezca una mejor localización de la litiasis, en la actualidad se emplean tanto la ecografía como la fluoroscopia.
La fragmentación de las litiasis por las ondas de choque se produce por la combinación de los siguientes mecanismos: por el estrés tensor y de cizallamiento, cavitación, compresión producida por el avance de la onda y por la fatiga dinámica de la litiasis al estar sometida a las ondas de choque ${ }^{3}$.

En nuestro estudio revisamos los resultados del tratamiento de 1500 pacientes con una única litiasis, localizada tanto a nivel renal como ureteral, manejada con un litotritor de última generación de ondas de choque electromagnéticas el Siemens Lithostar Modularis Uro Plus, para valorar la eficacia de este nuevo aparato.

\section{PACIENTES Y METODOS}

Entre junio de 2000 y diciembre de 2006, se han tratado con litotricia extracorpórea 1500 pacientes con litiasis única con localización renal o ureteral, los pacientes con litiasis múltiple han sido excluidos de este estudio. Los tratamientos se han efectuado en la Obra Hospitalaria Nuestra Señora de Regla de León, y se han efectuado por un único Urólogo. El aparato de litotricia empleado fue el "Lithostar Modularis UroPlus (Seimens Medical Systems, Erlangen, Alemania). Es un litotritor electromagnético de tercera generación con un emisor de ondas electromagnéticas (Electromagnetic Shock Wave C plus), integrado a un arco fluoroscópico en C (Siremobil Iso-C) que permite la localización de la litiasis con un movimiento de eje de rayos $\mathrm{X}$ de $-20^{\circ} \mathrm{a}+20^{\circ}$. Además tiene integrado un localizador ecográfico opcional externo, y una mesa de tratamiento endourológico (Modularis Uro), que facilita la aplicación de la litotricia. La profundidad de penetración de la onda de choque es de $14 \mathrm{~cm}$, la zona focal es de $80 \times 5 \mathrm{~mm}$, con un pico de energía de $101 \mathrm{~mJ}$.

El diagnóstico de los pacientes fue por métodos convencionales, urografía endovenosa, ecografía y/o TAC. El tamaño de la litiasis se determinó por el diámetro mayor de la litiasis, medido por radiografía simple de abdomen previa a comenzar el tratamiento. A todos los pacientes se les hizo estudio preoperatorio y recibieron sedación endovenosa con alfentanilo y dormicum, y fueron tratados de forma ambulatoria. La desintegración de la litiasis durante el proceso se comprobó mediante fluoroscopia, ecografía o ambas. A la semana del tratamiento se evaluó mediante radiografía simple y ecografía la presencia o ausencia de hematoma y la evolución de 
la litiasis. En nuestro servicio, usamos el siguiente protocolo: tratamiento de las litiasis "in situ", aplicando el número de sesiones necesario hasta obtener fragmentos menores de 2-3 mm o la eliminación de la litiasis, momento en el que se les da de alta. Sólo se coloca catéter doble pig-tail o nefrostomía a posteriori de la litotricia, en los casos en los que existen complicaciones y/o que existe una obstrucción de la vía urinaria, o en caso de impactación de la litiasis para su movilización. El tiempo medio de resolución, desde que los pacientes son vistos por primera vez en el Servicio hasta que son dados de alta, fue de unas 5 semanas. La aplicación de la energía empleada en las ondas electromagnéticas depende de la localización de las litiasis, con unos máximos de hasta el nivel 4 de energía (52 mJ ó 16 $\mathrm{kV}$ ) en las de localización renal, de hasta 6 en localización en uréter proximal (76 mJ ó $17,5 \mathrm{kV}$ ) y de hasta 8 en los de localización en uréter distal (101 mJ ó $19 \mathrm{kV}$ ). La frecuencia de aplicación fue de 6090 ondas/minuto en las localizaciones en riñones y uréter proximal y de 90/120 ondas/minuto en las de uréter distal. El número medio de ondas por sesión fue de 3.000-3.500.

La localización de la litiasis fue en 664 casos derecha $(44,2 \%)$ y en 836 izquierda $(55,7 \%)$. La edad media de los pacientes fue de 53,05+14,79 años (rango 15-89 años). 931 fueron varones 62,1\%) y 569 mujeres (37,9\%). El tamaño medio de la litiasis ha sido 1,309+0,88 cm (rango 0,4-9 cm) (Tabla 1). La localización fue en un 55,2\% ureteral y en un $44,8 \%$ renal (Tabla 2).

Tabla 1. Tamaño de las litiasis y porcentaje de distribución.

\begin{tabular}{lcc}
\hline Tamaño cm & Número & $\%$ \\
\hline$\leq 0,5$ & 159 & 10,6 \\
$>0,5<1$ & 110 & 7,3 \\
1 & 621 & 41,4 \\
$>1<2$ & 361 & 24,1 \\
2 & 111 & 7,4 \\
$\geq 2<3$ & 153 & 10,2 \\
$>2<3$ & 42 & 2,8 \\
$>3<4$ & 52 & 3,5 \\
$>4$ & 44 & 2,9 \\
\hline
\end{tabular}

Tabla 2. Localización de las litiasis y porcentaje de distribución por localización de las mismas.

\begin{tabular}{lccc}
\hline Localización & Número & \% Total & \% Parcial \\
\hline Total & 1500 & & \\
Ureteral & 828 & $55,2 \%$ & \\
U. lumbar & 420 & $28,5 \%$ & $51,7 \%$ \\
U. Iliaco & 71 & $4,7 \%$ & $8,6 \%$ \\
U. pélvico & 329 & $21,9 \%$ & $39,7 \%$ \\
Renal & 672 & $44,8 \%$ & \\
Cáliz Sup & 62 & $4,1 \%$ & $9,2 \%$ \\
Cáliz Med & 61 & 4,1 & $9,1 \%$ \\
Cáliz Inf & 204 & $13,6 \%$ & $30,4 \%$ \\
Pelvis & 345 & $23 \%$ & $51,3 \%$ \\
\hline
\end{tabular}

\section{RESULTADOS}

En los 1500 pacientes hemos precisado una media de sesiones por litiasis de 1,577 $\pm 1,091$ (con un rango de 1-10). El 66,9\% de los pacientes precisaron una sola sesión, el $86,5 \%$ precisaron menos de 2 sesiones y el 94,3\% menos de 3, solamente el 5\% de los pacientes precisaron 4 ó más sesiones para la fragmentación de la litiasis. Existen diferencias entre el número medio de sesiones de litotricia necesarias según el tamaño de la litiasis, en las litiasis menores de $1 \mathrm{~cm}$. la media de sesiones fue 1,2 , en las de $1 \mathrm{~cm}$ fue 1,3 , en las de $2 \mathrm{~cm}$ se precisaron 2 sesiones de media y el número fue ascendiendo al incrementar el tamaño. El número de pacientes libres de litiasis en una sola sesión ha disminuido progresivamente con el tamaño, desde más del 80\% en las litiasis menores de $1 \mathrm{~cm}$ al $5 \%$ en las litiasis de gran tamaño (Tabla 3).

Tabla 3. Media de sesiones de litotricia y porcentaje de pacientes resueltos con una y dos sesiones dependiendo del tamaño de las litiasis.

\begin{tabular}{lcccc}
\hline Tamaño & Número & Media sesiones & \% $\mathbf{1}^{\mathbf{a}}$ sesión & \% $\mathbf{2}^{\mathbf{a}}$ sesión \\
\hline$\leq 05$ & 159 & $1,157 \pm 0,457$ & 87,4 & 97,5 \\
$>0,5<1$ & 110 & $1,345 \pm 0,656$ & 74,5 & 91,8 \\
$<1$ & 269 & $1,234 \pm 0,554$ & 82,2 & 95,2 \\
1 & 621 & $1,348 \pm 0,717$ & 75,7 & 92,1 \\
$>1<2$ & 361 & $1,645 \pm 1,026$ & 60,4 & 85 \\
2 & 111 & $2,099 \pm 1,293$ & 41,4 & 70,3 \\
$>1 \leq 2$ & 472 & $1,752 \pm 1,110$ & 55,9 & 81,6 \\
$>2<3$ & 42 & $2,381 \pm 1,766$ & 40,5 & 69 \\
3 & 41 & $2,268 \pm 1,747$ & 46,3 & 70,7 \\
$>3<4$ & 11 & $2,909 \pm 2,809$ & 45,4 & 72,7 \\
$\geq 4<5$ & 24 & $2,667 \pm 1,786$ & 29,2 & 54,2 \\
$\geq 5$ & 20 & $4,050 \pm 2,350$ & 5 & 25 \\
\hline
\end{tabular}


La localización de las litiasis fue en un 55,2\% en el uréter, en especial en el uréter lumbar, y en un $44,8 \%$ en la unidad renal, la mitad en la pelvis y la siguiente en frecuencia en el cáliz inferior. La media de sesiones fue en las ureterales de 1,4 y en las renales de 1,7. El índice de retratamiento fue en las ureterales del $28 \%$ y en las renales del 39\%. Estos datos indican una mayor eficacia en las de localización ureteral, si bien también influye el menor tamaño de éstas (1,3 cm de media), respecto a las de localización renal (1,7 cm de media) (Tabla 4).

En el 22\% de los pacientes se precisó maniobras auxiliares durante el tratamiento de las litiasis, siendo más frecuentes estos procedimientos en las localizadas en el uréter, y dentro de estas en las de localización en uréter iliaco y pélvico, con un gran porcentaje de ureteroscopias. En las de litiasis de localización renal se precisaron maniobras en un $19 \%$, siendo generalmente estas un cateterismo, y más frecuentes en las de localización en pelvis renal, debido a su tamaño, para evitar complicaciones (Tabla 5).

Hemos obtenido una tasa de pacientes libres de litiasis o con restos menores de $3 \mathrm{~mm}$ en más de un 95\% en los de localización ureteral, y en un $80 \%$ en las de localización renal. En esta localización, el peor porcentaje es en los situados en el cáliz inferior, con un 23\% entre sedimentados y pequeños fragmentos susceptibles de expulsión (Tabla 6).

\section{DISCUSIÓN}

Desde la primera descripción del tratamiento de las litiasis urinarias mediante litotricia extracorpórea en $1982^{1,4}$, los litotritores han evolucionado intentando

Tabla 4. Tamaño, media de sesiones y porcentaje de litiasis resueltas en una y dos sesiones por localización de las litiasis.

\begin{tabular}{lccccc}
\hline Localización & Tamaño & Número & Media sesiones & \% $\mathbf{1}^{\mathbf{a}}$ sesión & \% 2 $\mathbf{~ s e s i o ́ n ~}^{\text {án }}$ \\
\hline Total & $1,309 \pm 0,88$ & 1500 & $1,577 \pm 1,091$ & 66,9 & 86,5 \\
Uréter & $1,01 \pm 0,38$ & 828 & $1,423 \pm 0,835$ & 71,7 & 90,9 \\
Lumbar & $1,02 \pm 0,37$ & 428 & $1,367 \pm 0,717$ & 72,9 & 93 \\
Iliaco & $1,2 \pm 0,41$ & 71 & $1,592 \pm 1,008$ & 66,2 & 83,1 \\
Pélvico & $0,96 \pm 0,37$ & 329 & $1,459 \pm 0,927$ & 71,4 & 90 \\
Renal & $1,67 \pm 1,15$ & 672 & $1,768 \pm 1,317$ & 61 & 81 \\
Cáliz sup. & $1,37 \pm 0,73$ & 62 & $1,387 \pm 1,014$ & 80,6 & 90,3 \\
Cáliz med. & $1,06 \pm 0,37$ & 61 & $1,41 \pm 0,824$ & 73,8 & 90,2 \\
Cáliz inf. & $1,29 \pm 0,63$ & 204 & $1,51 \pm 0,874$ & 66,7 & 87,3 \\
Pelvis & $2,07 \pm 1,37$ & 345 & $2,052 \pm 1,569$ & 51,9 & 73,9 \\
\hline
\end{tabular}

Tabla 5. Maniobras auxiliares y tipos en porcentaje en las distintas localizaciones de las litiasis.

\begin{tabular}{|c|c|c|c|c|c|c|c|c|}
\hline Localización & Maniobras & Catéter & URS & Nefrost. & Ureterolit. & PCN & Abierto & Cistosc. \\
\hline Total & 21,9 & 11,7 & 10,3 & 3,2 & 0,2 & 0,1 & 0,1 & 0,1 \\
\hline Uréter & 24,5 & 10,1 & 14,7 & 4 & 0,2 & 0,2 & & 0,2 \\
\hline Lumbar & 20,8 & 12,6 & 7,7 & 4,4 & 0,5 & 0,5 & & \\
\hline Iliaco & 28,2 & 15,5 & 19,7 & 8,4 & & & & \\
\hline Pélvico & 28,6 & 5,5 & 23 & 2,4 & 0,3 & & & 0,6 \\
\hline Renal & 18,8 & 13,7 & 4,9 & 2,2 & 0,1 & & 0,1 & \\
\hline Cáliz sup. & 11,3 & 8,1 & 3,2 & & & & & \\
\hline Cáliz med. & 6,5 & 4 & 1 & & & & & \\
\hline Cáliz inf. & 7,8 & 6,4 & 2 & & & & & \\
\hline Pelvis renal & 29 & 20,6 & 7,8 & 4,9 & 0,3 & & 0,3 & \\
\hline
\end{tabular}


Tabla 6. Porcentajes de resolución en los distintas localizaciones de las litiasis

\begin{tabular}{lcccc}
\hline Tamaño & No restos & No efizaz & Expulsables & Sedimentado \\
\hline Total & 91,3 & 1 & 2,8 & 4,9 \\
Uréter & 97,7 & 1,7 & 0,5 & 0,1 \\
Lumbar & 98,6 & 0,5 & 0,7 & 0,2 \\
Iliaco & 100 & & & \\
Pélvico & 96 & 3,6 & 0,3 & \\
Renal & 82,6 & 0,1 & 5,7 & 10,9 \\
Cáliz sup. & 83,9 & 1,6 & 3,2 & 11,3 \\
Cáliz med. & 83,6 & & 8,2 & 8,2 \\
Cáliz inf. & 77,4 & & 11,3 & 11,3 \\
Pelvis renal & 87 & & 2,3 & 10,7 \\
\hline
\end{tabular}

una mayor eficacia, con una menor morbilidad y menor coste. En un principio la energía empleada era electrohidraúlica, en la actualidad se prefiere las ondas electromagnéticas debido a que son más fácilmente controlables y la cantidad de energía más constante y produce menos dolor en el paciente debido a que entra por un área mayor en el cuerpo del paciente. Dentro de las desventajas de las ondas electromagnéticas está que producen una alta concentración de energía en una región focal muy pequeña, favoreciendo la aparición de lesiones renales tipo hematomas, pudiendo llegar hasta tasas del $4 \%{ }^{6}$, en el caso del litotritor Lithostar modularis el área focal se ha aumentado para disminuir este tipo de complicaciones, presentando un área focal de 80 x $5 \mathrm{~mm}$. El Lithostar modularis de Siemens parece proveer un equilibrio entre las necesidades anestésicas y la tasa de eficacia en la fragmentación de las litiasis. La penetración de la energía hasta $14 \mathrm{~cm}$ favorece la focalización de las ondas de choque en la litiasis, en especial en los obesos. Además la mayor penetración aumenta la eficacia del tratamiento ${ }^{7}$ creando un foco activo mayor para la destrucción de las litiasis al incrementar la energía de las ondas ${ }^{8}$.

El número de sesiones de litotricia que hemos precisado en nuestro Centro para resolver las litiasis ha sido 1,57 sesiones por litiasis, es un alto porcentaje comparado con otras series ${ }^{5,9}$, en los que oscilan entre 1,2 y 1,4 tratamientos por pacientes. Nuestra serie se diferencia de dichas series en el tamaño de la litiasis, con una media de $1,3 \mathrm{~cm}$, mientras que en las otras series el tamaño medio es menor de $1 \mathrm{~cm}$. Otra diferencia es el porcentaje libre de litiasis en los otros trabajos, en ellas los pacientes libres de litiasis variaron de 68 a $86 \%$, en nuestra serie el porcentaje sin litiasis fue del 91\%. El porcentaje de retratamiento en nuestra serie fue del $33 \%$, también superior a las series anteriores, Esto indica una mayor eficacia en nuestra serie conseguida con un aumento del número de tratamientos. El porcentaje de éxitos de pacientes sin litiasis nos acerca al porcentaje del litotritor de mayor eficacia, el $\mathrm{HM} 3^{10}$, pero con menor número de complicaciones, menos anestesia, el tratamiento de pacientes de forma ambulatoria, pero con el inconveniente de mayor número de sesiones.

En nuestro estudio hemos observado la existencia de factores que influencian el número de sesiones, por un lado, el tamaño parece ser el principal determinante, al aumentar el tamaño aumenta el número de sesiones necesaria (Tabla 3). El tamaño puede influir en el mayor número de sesiones precisadas en nuestra serie, al ser la serie con el tamaño medio mayor de las litiasis. Otro factor que influye en este número es la localización de la litiasis, se precisan menos sesiones en los pacientes con localización ureteral que en la renal, existiendo incluso diferencias en cuanto a la localización tanto en el uréter como en el riñón (Tabla 4).

El porcentaje de pacientes que precisaron maniobras auxiliares durante el tratamiento fue del $22 \%$, este es un porcentaje alto, asociado al mayor tamaño de las litiasis que en las otras series. En el caso de las litiasis ureterales el porcentaje es mayor, próximo al $25 \%$, con un alto porcentaje de ureteroscopias, por encima del 14\%, lo cual contribuye a presentar un alto índice de eficacia, con más del 95\% de las litiasis resueltas, con ausencia de restos al combinar litotricia con maniobras complementarias. En el caso de las litiasis renales el porcentaje de maniobras es menor, del 18,8\%, y se debe principalmente a cateterismo debido al tamaño de las litiasis, aunque es de destacar que en las litiasis mayores de $3 \mathrm{~cm}$ de diámetro mayor, el porcentaje de cateterismos es inferior al 35\%, indicando la no necesidad de colocación de catéteres ureterales a los pacientes previamente a la litotricia para evitar complicaciones, lo cual supone un gran ahorro en material. Este alto porcentaje de maniobras hace que el cociente de eficacia EQ) ${ }^{11}$, descrito para comparación de distintos litotritores haya disminuido 
un poco, pero se mantiene entre $\mathrm{EQ}=0,55$ de las litiasis ureterales y el 0,68 de las litiasis renales, lo cual es muy próximo a las descritas en la literatura, e incluso muy similar al 0,65 descrito para el HM3 ${ }^{12}$.

\section{CONCLUSIONES}

Nuestra experiencia con el Lithostar modularis uro-plus muestra que este litotritor es seguro, y aunque presentamos una media de sesiones ligeramente superior a la media, esto nos permite obtener unos muy buenos resultados, con un alto porcentaje de pacientes sin litiasis, próximos al 90\%, presentando además un buen balance entre el la anestesia que se precisa y la fragmentación obtenida, pudiendo tratar a los pacientes de forma ambulatoria. Además, los resultados son similares a los reportados por HM3 aunque usando menos energía y precisando menos anestesia, indicando que los litotritores emisores de ondas electromagnéticas se convertirán con toda probabilidad en el estándar de litotricia en el futuro.

\section{REFERENCIAS}

1. Chaussy C, Schmiedt E Jockham D, Brendel W, Forssmann B, Walter V. First clinical experience with extracorporeally induced destruction of kidney stones by shock waves. J Urol. 1982;127 (3):417-420.

2. Teichman JM, Portis AJ, Cecconi PP, Bub WL, Endicott RC, Denes B, et al. In Vitro Comparison Of Shock Wave Lithotripsy Machines. J Urol. 2000;164(4):1259-1264.

3. Rassweiler JJ, Taillo GG, Chaussy C. Progress in Lithotriptor Technology EUA Update Series 3. 2005;17-36.
4. Chaussy C, Schüller J, Schmiedt E, Brandl H, Jocham D, Liedl B. Extracorporeal Shock Wave Lithotripsy (ESWL) For Treatment Of Urolithiasis. Urology. 1984;23(5 Spec No):59-66. 5. Heretis J, DAskalopoulos G, Delibaltidis O, Kalivianakis D, Papadimitrioy V, Sofras F. First Clinical Experience with Doli S 220F-XP Extracorporeal Lithotripter for Urinary Lithiasis. J Endourology. 2006;20(7):479-482.

6. Streem SB, Bata D, Tornito J, Karafa MT. A Multivariate Análisis Of Risk Factors Associated With Subcapsular Hematoma Formation Following Electromagnetic Shock Wave Lithotripsy. J Urol. 2004; 172:2271-2274.

7. Lingeman JE. Extracorporeal Shock Wave Lithotripsy: What Happened?. J Urol. 2003;169(1):63.

8. Granz B, Lanski M, Nanke B, Mahler M, Rohwedder A, Chausy G, Thüroff S. Lithostar Modularis: A Proven System With New, Innovative Features. Electromedica. 2003;71:53-57.

9. Egilmez T, Tekin MI, Gonen M, Kilinc F, goren R, Ozkardes H. Efficacy and safety of a new-generation shockwave lithotripsy machine in the treatment of single renal or ureteral stones: Experience with 2670 patients. J.Endourology. 2007;21(1):2327.

10. Lingeman JE, Newman D, Mertz JHO, Mosbaugh PG Steele RE, KAhnosski RJ, et al. Extracorporeal Shock Wave Lithotripsy. The Methodist Hospital Of Indiana Experience. J Urol. 1986:1134-1137.

11. Denstedt JD, Clayman RV, Preminger GM. Efficiency Quotient As A Means Of Comparing Lithotripters (Abstract) J Endourology 1990; 4 (suppl): 100

12. Cass AS. Comparison Of Firest Generation (Dornier HM3) And Second Generation (Medstone STS) Lithotriptors: Treatment Results With 13,864 Renal And Ureteral Calculi. J Urol. 1995; 153(3 Pt 1):588-592.

Correspondencia autor: Dr. A. Vega Vega Servicio de Urología.

Complejo Hospitalario de León E-mail autor: debgarcia100@hotmail.com Información artículo: Original - Litiasis

Terabajo recibido: septiembre 2007 Trabajo aceptado: noviembre 2007 